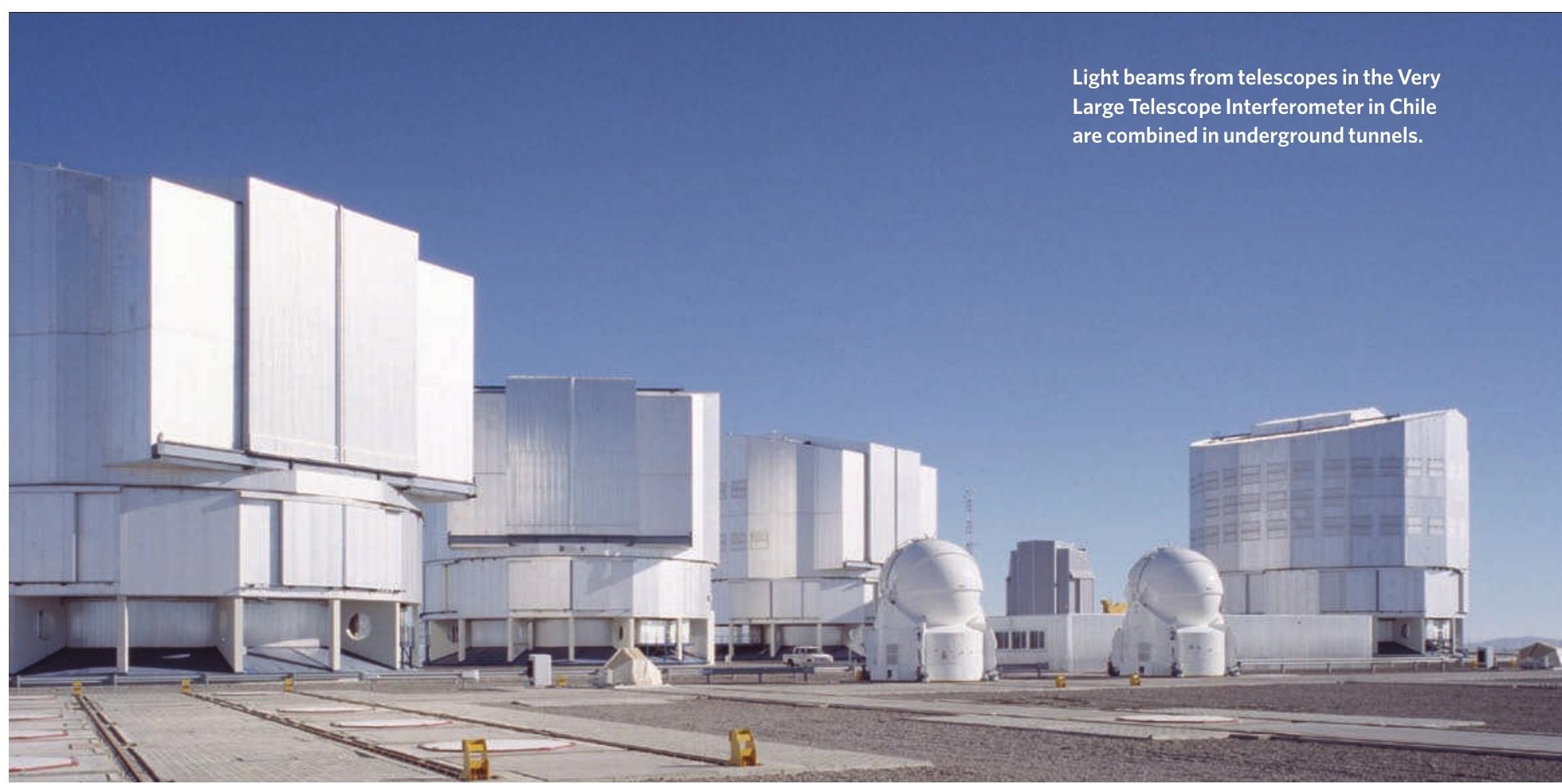

\title{
Telescope arrays give fine view of stars
}

\section{Optical interferometry is no longer on the fringe of astronomy.}

Overlooking Los Angeles, six small domes nestle amid the pine trees atop Mount Wilson. Individually, the 1-metre telescopes inside those buildings have no chance of competing with the biggest ground and space telescopes. But collectively, the Mount Wilson telescopes are producing some of the sharpest images ever made.

Spread in a Y-shaped array across the top of the mountain, the telescopes are part of the Center for High Angular Resolution Astronomy (CHARA). The light from each one is funnelled through vacuum tubes to a central shed, where it is combined in

optical interferometry has come of age. Several observatories are producing strong scientific results, including one reported on page 870 by researchers using the CHARA array ${ }^{1}$. At the end of last year, the team imaged a disk of dust almost as wide as the Solar System as it crept in front of a large, old star and blotted out its light. This was the first direct image of an eclipsing binary system that has puzzled astronomers "This is moving us into a realm that radio astronomy has been able to enjoy for decades." for more than a century. "This is moving us into a realm that radio astronomy has been able to enjoy for decades," says Robert Stencel, an astronomer at the University of Denver in Colorado and a coauthor on the paper.

a process called interferometry. Merging the light beams from the widely separated domes gives CHARA a resolving power, or sharpness, equivalent to a single telescope with a 330 metre mirror. That's more than 50 times better than the Hubble Space Telescope's resolution, allowing CHARA to see details on the surfaces of stars where other telescopes just see blurry blobs of light.

Radio astronomers have relied on interferometry for more than half a century, but optical astronomers have lagged behind. Now,
From the start, radio astronomers have enjoyed several advantages over optical observers. Earth's atmosphere doesn't blur radio waves as it does the shorter wavelengths of light. Moreover, radio signals gathered at separate dishes can be digitized, transmitted electronically, then recombined into an interference pattern - the basis of a high-resolution image. This ease of handling has allowed radio astronomers to amalgamate data from dishes all over the globe, creating virtual arrays with baselines as wide as Earth itself.
But with optical interferometry, astronomers must intertwine the faint light beams in real time by routing them through tunnels with nanometre-level precision. They also have to counteract the effects of atmospheric blurring using a complex technology called adaptive optics. And because many optical arrays use relatively small telescopes, they have trouble gathering enough light to study anything but bright stars nearby.

\section{Technical advances}

Even with those constraints, optical interferometry has yielded new insights about stars, such as how binary systems swap mass and how stars bulge when they spin. Now, astronomers are pushing the technique by combining light from more than two telescopes. Multiple beams not only make data collection more efficient - more photons are caught and used - they also provide cross-checks on the data, making it easier to build up an image from the interference pattern. CHARA first demonstrated ${ }^{2}$ a four-beam combiner in 2007, and next year it plans to try for a record six beams at once.

The advances are turning once-difficult experiments into more routine operations, opening up the process to astronomers who are not experts in interferometry. "Now we're 
getting more general users," says Françoise Delplancke, head of the interferometry group for the European Southern Observatory's Very Large Telescope Interferometer (VLTI) in Chile. The number of science papers based on optical interferometry has surged as well, from 9 in 1999 to 56 last year. The VLTI, the focus of European support, is responsible for about half of those.

The support for US facilities is more fragmented. CHARA is a university-run operation supported by the National Science Foundation. A potential rival, the Magdalena Ridge Observatory in New Mexico, has run into delays because of funding problems. A NASA-supported interferometer involving the twin 10-metre Keck telescopes in Hawaii was supposed to achieve VLTI-like capabilities with the addition of four to six small 'outrigger' telescopes. But the auxiliary project was derailed in 2006 over environmental and cultural concerns about building new telescopes on the Mauna Kea summit.

Yet not everyone has given up on Mauna Kea, which holds the largest concentration of huge telescopes on Earth. Guy Perrin, an astronomer at the Paris Observatory and principal investigator for the Optical Hawaiian Array for Nano-radian Astronomy (OHANA), is connecting the seven large telescopes at the summit into an array with a baseline of 800 metres. As a proof of principle, Perrin has already combined light from the two Keck telescopes via inconspicuous optical fibres, which would obviate the need to connect the telescopes with tunnels ${ }^{3}$.

Reached by telephone atop the summit, Perrin last week was busy implementing a second stage - a fibre-optic link to connect the Gemini North telescope to the CanadaFrance-Hawaii Telescope. Down in Chile, at the VLTI, he is helping to develop integrated optics, which would combine beams efficiently on tiny silicon chips rather than in large, complicated rooms.

Although the technological hurdles to the OHANA project are still high, Perrin says that a bigger problem could be getting all the Mauna Kea observatories to simultaneously offer up their telescope time - a precious and fiercely guarded resource. "It will be easier to convince the communities that are behind the telescopes," says Perrin, "if we first demonstrate that interferometry is a big player in science today."

Eric Hand

1. Kloppenborg, B. et al. Nature 464, 870-872 (2010)

2. Monnier, J. D. et al. Science 317, 342-345 (2007).

3. Perrin, G. et al. Science 311, 194 (2006).

\section{Charities warm to climate}

to commercial investment in clean energy

Global steps to battle climate change might have faltered, but philanthropic institutions in the United States have swung into action, more than tripling their support for climaterelated causes in 2008. Donations jumped from the 2007 total of US $\$ 240$ million to \$897 million in 2008 (see 'Climate concern'), according to a report from the Foundation Center, an organization that supports philanthropies, in New York.

The funding is going to a range of activities, including efforts to reduce greenhouse-gas emissions and to prepare cities for warmer temperatures and higher sea levels. Foundation money is also supporting academic researchers studying the effects of climate change and ways to reduce pollution. In 2008, for example, the Rockefeller Foundation in New York gave a grant to Stanford University in California for studies on how agriculture could adapt to a changing climate. The ClimateWorks Foundation in San Francisco, California, is supporting research around the world, including a grant to Wang Lan, a materials scientist at the China Building Materials Academy in Beijing, who is working to reduce greenhouse-gas emissions from cement production.

The vast majority of the increase in 2008 came from the William and Flora Hewlett Foundation in Menlo Park, California, which gave a total of \$549 million. Hewlett's donations included a one-time contribution of $\$ 500$ million to ClimateWorks, which aims to help countries limit carbon dioxide concentrations in the atmosphere to less than 450 parts per million.

Many other foundations also bumped up their spending. All told, 267 foundations other than Hewlett distributed 1,578 grants for climate change, representing a $45 \%$ increase in their giving compared with 2007, according to the Foundation Center report, which is entitled Climate Change: The U.S. Foundation Response.

A generational change may account for part of the sudden generosity. Baby boomers are showing more concern about climate change than previous generations did, says Rachel Leon, executive director of the Environmental Grantmakers Association in New York, a trade group of environmentally focused foundations. These people are now starting to set up their own foundations with a strong emphasis on climate change.

The efforts of the foundations pale next
- $\$ 173$ billion in 2008 and $\$ 162$ billion last year, according to market analysts Bloomberg New Energy Finance in London. But foundations can fund projects regardless of their potential pay-off, says Ethan Zindler, the company's head of US research. "They view it as a social imperative," he says. ClimateWorks, for example, collaborates with smaller foundations around the world on projects including the development of vehicle-fuel standards in India and appliance standards in China.

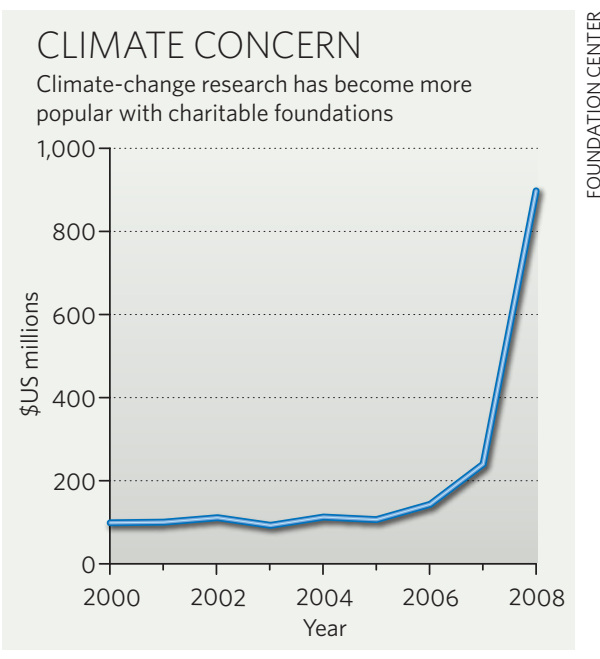

Other efforts aim to help developing countries adapt to change. Under a fiveyear, \$70-million commitment in 2007, Rockefeller established the Asian Cities Climate Change Resilience Network, which focuses on aiding smaller cities, such as Surat in India, make growth decisions that help them survive a shifting climate. "We are not really an environmental foundation but a poverty-reduction foundation. But we see a connection between them," says Cristina Rumbaitis del Rio, an associate director at the Rockefeller Foundation.

None of the foundations contacted by Nature would say what it plans to give in 2010. Because Hewlett will not repeat its \$500-million, one-time donation, the total foundation support for climate-related causes is likely to drop from its 2008 high, but Steven Lawrence, the director of research for the Foundation Center and the author of the new report, expects funding this year to surpass the 2007 amount. "My expectation is to continue to see growth in giving." Laura Thompson Osuri 